\title{
Combating entanglement sudden death with non-local quantum-error correction
}

\author{
Isabel Sainz and Gunnar Björk \\ School of Information and Communication Technology, \\ Royal Institute of Technology (KTH), \\ Electrum 229, SE-164 40 Kista, Sweden
}

(Dated: November 6, 2018)

\begin{abstract}
We study the possibility of preventing finite-time disentanglement caused by dissipation by making use of non-local quantum error correction. This is made in comparison of previous results, where was shown that local quantum error correction can delay disentanglement, but can also cause entanglement sudden death when is not originally present.
\end{abstract}

\section{INTRODUCTION}

In the last decade substantial attention has been devoted to entanglement because it is considered to be the physical resource on which quantum technologies are based [1]. For this reason, entanglement dynamics have been widely studied, with the goal of finding ways to manipulate entangled states in a practical way, and in addition, to get a fundamental understanding of entanglement and all its embodiments.

When interacting with a reservoir the fragile quantum states are easily destroyed. E.g., several years ago it was shown that a pair of initially entangled qubits can loss their entanglement in a finite time and not asymptotically as one would naively expect [2]. This phenomena was eventually called entanglement sudden death [3] (ESD) and several investigations have followed, see [3]- 77]. Recently ESD was demonstrated in a linear optics experiment [8].

Several schemes have been proposed in order to preserve fragile quantum states when interacting with a reservoir, for example decoherence-free subspaces [9] and quantum error correction (QEC) (see [10]-[19]). A recent worry expressed in [7] is that if an entangled state suffers a "sudden death" the entanglement is irrevocably lost, and therefore no QEC scheme can help to restore the state.

Recently, using the $[4,1]$ code which encodes a single qubit into four physical qubits and 
which was specifically constructed to deal with an amplitude damping channel [17], it has been demonstrated that when a pair of initially entangled qubits locally encoded with a $[4,1] \times[4,1]$ code, and subjected to local recovery operations similar to the ones proposed in [19], QEC can delay ESD in some cases, but may cause ESD for certain states that will not succumb to ESD without QEC [20]. We would like to stress that by local we mean that each qubit is encoded, measured and recovered separately. In the same work [20], it is mentioned that even using the non-local code $[6,2]$ which encodes a pair of logical qubits into six physical qubits [19], the obtained results does not differ qualitatively from the shown in [20].

In this work we demonstrate this explicitly, which means that even when the QEC protocol is non-local, in all the stages, there is not a qualitatively difference with the local code studied before [20]. We also find that the success of non-local coding and error recovery has a rather substantial state dependence.

\section{THE MODEL}

As a simple example we will consider an amplitude damping channel, that for one qubit can be represented in terms of the Kraus operators :

$$
\hat{E}_{0}=\left(\begin{array}{cc}
1 & 0 \\
0 & \sqrt{1-\gamma}
\end{array}\right), \quad \hat{E}_{1}=\left(\begin{array}{cc}
0 & \sqrt{\gamma} \\
0 & 0
\end{array}\right),
$$

where $\gamma$ is the jump probability from the excited $(|1\rangle)$ to the ground $(|0\rangle)$ state, $\hat{E}_{0}$ is the one qubit no-jump operator that leaves the ground state unchanged, but decreases the excited state probability with the factor $1-\gamma$, while $\hat{E}_{1}$ represents the jump operator that transforms the state $|1\rangle$ into the state $|0\rangle$ with probability $\gamma$.

To study the possibility of protecting a two qubit state and particularly entanglement by non-local coding, where the pair of qubits are encoded together, and to compare it with previous results obtained by local coding [20], we will use the [6,2] code introduced in [19]. This code is specially made for protecting two qubits against this kind of disturbance. It encodes an arbitrary two-qubit pure state

$$
\left|\varphi_{0}\right\rangle=\cos \alpha \cos \delta|11\rangle+\sin \alpha \cos \delta e^{i \epsilon_{1}}|00\rangle+\cos \beta \sin \delta e^{i \epsilon_{2}}|10\rangle+\sin \beta \sin \delta e^{i \epsilon_{3}}|01\rangle
$$


into the corresponding logical state

$$
\begin{aligned}
\left|\varphi_{0}\right\rangle_{L}= & \cos \alpha \cos \delta|11\rangle_{L}+\sin \alpha \cos \delta e^{i \epsilon_{1}}|00\rangle_{L} \\
& +\cos \beta \sin \delta e^{i \epsilon_{2}}|10\rangle_{L}+\sin \beta \sin \delta e^{i \epsilon_{3}}|01\rangle_{L},
\end{aligned}
$$

were the codewords $|00\rangle_{L},|01\rangle_{L},|10\rangle_{L}$ and $|11\rangle_{L}$, are given by, [19]

$$
\begin{aligned}
|00\rangle_{L} & =(|000000\rangle+|111111\rangle) / \sqrt{2}, \\
|01\rangle_{L} & =(|001001\rangle+|110110\rangle) / \sqrt{2}, \\
|10\rangle_{L} & =(|000110\rangle+|111001\rangle) / \sqrt{2}, \\
|11\rangle_{L} & =(|110000\rangle+|001111\rangle) / \sqrt{2} .
\end{aligned}
$$

Above, we have used the notation $|000000\rangle=|0\rangle \otimes \ldots \otimes|0\rangle$, etc. It is worth noticing that the codewords (2)-(5) in principle can be mapped in any way onto the physical states on the right-hand side. With different mappings we will obtain different results for a given state $\left|\varphi_{0}\right\rangle$, where the labeling of the physical state $(|000000\rangle+|111111\rangle) / \sqrt{2}$ with the possible codewords $|i j\rangle_{L}, i, j=0,1$ is the main cause of such differences.

We will suppose that every physical qubit is interacting with its own environment, a situation that leads to ESD (see [2]-[7] and references therein). This implies that the amplitude damping channels can be considered to be independent, which means that the many-qubit amplitude-damping channel will be described by the tensorial product of the corresponding Kraus operators as in [20]. For simplicity we will also assume that all the qubits are damped with the same probability $\gamma$.

As was pointed out in [19], the one physical-qubit damping-errors are spanned by orthogonal subspaces. Nevertheless, in this channel we have conditional evolution (the no-damping error introduces some distortion in the original state). However, the distortion generated by this channel in this code is of the order of $\gamma^{2}$, so it satisfies the approximate QEC conditions [17]. Therefore the no-damping evolution is "approximately" spanned by the codewords (2)(5). Every one of the subspaces related to no-damping and one-qubit damping are spanned by four 64-dimensional vectors $\left\{\left|R_{k 00}\right\rangle,\left|R_{k 01}\right\rangle,\left|R_{k 10}\right\rangle,\left|R_{k 11}\right\rangle\right\}$, where $k=0$ denotes no damping, $k=1, \ldots, 6$ means damping in the first to the sixth physical qubit respectively, and the last two subscripts $(i j)$ in each $\left|R_{k i j}\right\rangle$ refer to the corresponding two-qubit state. In total we have 28 vectors spanning the no-damping and one-qubit damping. For no damping 
we have the codewords (2) -(5),

$$
\left|R_{000}\right\rangle=|00\rangle_{L}, \quad\left|R_{001}\right\rangle=|01\rangle_{L}, \quad\left|R_{010}\right\rangle=|10\rangle_{L}, \quad\left|R_{011}\right\rangle=|11\rangle_{L} .
$$

Meanwhile, for the one-qubit damping one obtains

$$
\begin{array}{llll}
\left|R_{100}\right\rangle=|011111\rangle & \left|R_{101}\right\rangle=|010110\rangle & \left|R_{110}\right\rangle=|011001\rangle & \left|R_{111}\right\rangle=|010000\rangle \\
\left|R_{200}\right\rangle=|101111\rangle & \left|R_{201}\right\rangle=|100110\rangle & \left|R_{210}\right\rangle=|101001\rangle & \left|R_{211}\right\rangle=|100000\rangle \\
\left|R_{300}\right\rangle=|110111\rangle & \left|R_{301}\right\rangle=|000001\rangle & \left|R_{310}\right\rangle=|110001\rangle & \left|R_{311}\right\rangle=|000111\rangle, \\
\left|R_{400}\right\rangle=|111011\rangle & \left|R_{401}\right\rangle=|110010\rangle & \left|R_{410}\right\rangle=|000010\rangle & \left|R_{411}\right\rangle=|001011\rangle \\
\left|R_{500}\right\rangle=|111101\rangle & \left|R_{501}\right\rangle=|110100\rangle & \left|R_{510}\right\rangle=|000100\rangle & \left|R_{511}\right\rangle=|001101\rangle \\
\left|R_{600}\right\rangle=|111110\rangle & \left|R_{601}\right\rangle=|001000\rangle & \left|R_{610}\right\rangle=|111000\rangle & \left|R_{611}\right\rangle=|001110\rangle
\end{array}
$$

It is worth noticing that the vectors given above are 28 basis vectors out of 64 . The rest of the basis vectors can be found in the following manner: first we should add vectors similar to (2) -(5), but with a minus instead of a plus. For the rest of the basis' elements there are several ways. For example, we can add the rest of the vectors of the computational basis with three excitations and, we can arrange the vectors of the computational basis with two and four excitations not considered in (2)-(5), in a similar way as in (2) -(5) and consider not only the addition, but the substraction too. However, to list one or several of the complete bases lies outside the scope of this paper.

In order to make the syndrome measurement more transparent, let us introduce the following unitary transformation, that changes the basis given above to the computational one

$$
\hat{S}=\sum_{k=0}^{15} \sum_{i, j=0}^{1}|i j \operatorname{Bin}(k)\rangle\left\langle R_{k i j}\right|,
$$

where the $\operatorname{Bin}(k)$ is the binary representation of the $k=0, \ldots, 15$ for the last four qubits. The error syndrome detection is completed when we measure the last four (physical) qubits, i.e., $\operatorname{Bin}(k)$. This allows us to detect possible errors in a way that preserves any superposition in the two first qubits. When measuring $\operatorname{Bin}(k)$, if the outcome is, for example 0000 (see Table I), we know that no qubit was damped. If, on the other hand, the results are $0001,0010,0011,0100,0101$ or 0110 we conclude that there is a jump in the qubit one to six, respectively. It is important to notice that this assumption is only true until a certain "degree" $\sim \gamma^{2}$, because more than one-qubit damping could lead the same state as one qubit 
TABLE I: Syndrome outcomes and corresponding recovery operations.

\begin{tabular}{|c|c|}
\hline Syndrome & Recovery operation \\
\hline $\operatorname{Bin}(0)=0000$ & $\hat{R}_{0}=\sum_{i, j=0}^{1}|i j\rangle_{L}\langle i j \operatorname{Bin}(0)|$ \\
$\operatorname{Bin}(1)=0001$ & $\hat{R}_{1}=\sum_{i, j=0}^{1}|i j\rangle_{L}\langle i j \operatorname{Bin}(1)|$ \\
$\operatorname{Bin}(2)=0010$ & $\hat{R}_{2}=\sum_{i, j=0}^{1}|i j\rangle_{L}\langle i j \operatorname{Bin}(2)|$ \\
$\operatorname{Bin}(3)=0011$ & $\hat{R}_{3}=\sum_{i, j=0}^{1}|i j\rangle_{L}\langle i j \operatorname{Bin}(3)|$ \\
$\operatorname{Bin}(4)=0100$ & $\hat{R}_{4}=\sum_{i, j=0}^{1}|i j\rangle_{L}\langle i j \operatorname{Bin}(4)|$ \\
$\operatorname{Bin}(5)=0101$ & $\hat{R}_{5}=\sum_{i, j=0}^{1}|i j\rangle_{L}\langle i j \operatorname{Bin}(5)|$ \\
$\operatorname{Bin}(6)=0110$ & $\hat{R}_{6}=\sum_{i, j=0}^{1}|i j\rangle_{L}\langle i j \operatorname{Bin}(6)|$ \\
$\operatorname{Bin}(7)=0111$ & Measure the first two qubits \\
$\operatorname{Bin}(8)=1000$ & and project onto $\hat{I}_{L} / 4$ \\
$\operatorname{Bin}(9)=1001$ & \\
$\operatorname{Bin}(10)=1010$ & \\
$\operatorname{Bin}(11)=1011$ & \\
$\operatorname{Bin}(12)=1100$ & \\
$\operatorname{Bin}(13)=1101$ & \\
$\operatorname{Bin}(14)=1110$ & \\
$\operatorname{Bin}(15)=1111$ & \\
\hline
\end{tabular}

damping. Similarly, some two or four-qubit damping could lead to the state $|000000\rangle$, which is part of the no-damping basis state.

Once that we have measured the syndrome we should apply a proper recovery operation, summarized in Table II If the outcome is " $\operatorname{Bin}(k)$ " for $k=0, \ldots, 6$ we will preserve the superpositions between the two first qubits by applying the operation $\hat{R}_{k}$. If we measure "Bin $(k)$ " for $k=7, \ldots, 15$ we know that more than one qubit was damped, and we cannot correct the state, but we will try to keep some features of the original state by projecting the system to the state $\hat{I}_{L} / 4$ for reasons of symmetry, where $\hat{I}_{L}=|00\rangle_{L}\left\langle\left. 00\right|_{L}+\mid 01\right\rangle_{L}\left\langle\left. 01\right|_{L}+\right.$ $|10\rangle_{L}\left\langle\left. 10\right|_{L}+\mid 11\right\rangle_{L}\left\langle\left. 11\right|_{L}\right.$ is the identity in the codeword space. In this scheme, it is always possible to reconstruct a two-qubit density matrix from the six-qubit recovered state, because 
the state after recovering is in the four-dimensional codeword sub-space, which enables us to compute (measure) not only the exact fidelity but the pairwise entanglement by means of the standard concurrence.

\section{ENTANGLEMENT SUDDEN DEATH AND QUANTUM ERROR CORRECTION}

As was pointed out in the Introduction, ESD is present in different scenarios for some bipartite states. In this context the so-called $X$-states [5] have been widely studied [6]. They have the property that when interacting with independent environments, the corresponding composite density matrix preserves the $X$ form in the computational basis. They also merit studying because they include the Bell-states and the Werner-states. Furthermore, among them one can find states that are subject to ESD and states that are not, when evolving under dissipation. Particularly, we will work with the Bell-like states of the form

$$
\begin{gathered}
\left|\phi_{0}\right\rangle=\cos \alpha|11\rangle+e^{i \beta} \sin \alpha|00\rangle, \\
\left|\psi_{0}\right\rangle=\cos \alpha|10\rangle+e^{i \beta} \sin \alpha|01\rangle .
\end{gathered}
$$

It is worth noticing that state $\left|\phi_{0}\right\rangle$ will succumb to ESD for $\alpha$ such that $|\tan \alpha|<1$, this was experimentally shown [8], meanwhile $\left|\psi_{0}\right\rangle$ will not become disentangled for any finite amount of dissipation. In order to show the effect of different mappings of the codeword space we will also consider the separable states

$$
\begin{aligned}
& \left|\zeta_{0}\right\rangle=\cos \alpha|01\rangle+e^{i \beta} \sin \alpha|00\rangle, \\
& \left|\xi_{0}\right\rangle=\cos \alpha|11\rangle+e^{i \beta} \sin \alpha|10\rangle .
\end{aligned}
$$

We will try to protect the information carried by the states (8)-(11) by encoding these states using the codewords given by the set of equations (2)-(诘), so that the corresponding logical states are

$$
\begin{aligned}
\left|\phi_{0}\right\rangle_{L} & =\cos \alpha|11\rangle_{L}+e^{i \beta} \sin \alpha|00\rangle_{L}, \\
\left|\psi_{0}\right\rangle_{L} & =\cos \alpha|10\rangle_{L}+e^{i \beta} \sin \alpha|01\rangle_{L}, \\
\left|\zeta_{0}\right\rangle_{L} & =\cos \alpha|01\rangle_{L}+e^{i \beta} \sin \alpha|00\rangle_{L}, \\
\left|\xi_{0}\right\rangle_{L} & =\cos \alpha|11\rangle_{L}+e^{i \beta} \sin \alpha|10\rangle_{L} .
\end{aligned}
$$


The standard way to quantify the efficiency of quantum information processes and protocols, particularly QEC is the fidelity, that for an initially pure state is just the overlap between the original/desirable state $\left|\varphi_{0}\right\rangle$ and the obtained one $\hat{\rho}$ after dissipation and application of the considered protocol. That is $\mathcal{F}=\left\langle\varphi_{0}|\hat{\rho}| \varphi_{0}\right\rangle$. In this case the fidelities between the initial encoded states (12)-(14) and the corresponding ones after amplitude damping modeled by the Kraus operators and QEC are given by

$$
\begin{aligned}
& \mathcal{F}_{\phi}=\mathcal{F}_{\zeta}=1-\frac{\gamma^{2}}{4}\left(21-9 \cos 2 \alpha-\sin ^{2} 2 \alpha \cos ^{2} \beta\right)+O\left(\gamma^{3}\right) \\
& \mathcal{F}_{\psi}=\mathcal{F}_{\xi}=1-\frac{\gamma^{2}}{4}\left(12-\sin ^{2} 2 \alpha \cos ^{2} \beta\right)+O\left(\gamma^{3}\right) .
\end{aligned}
$$

Here, the non-locality and the codeword labeling is manifested in the fact that the fidelities when considering the initial states $\left|\phi_{0}\right\rangle_{L}$ and $\left|\zeta_{0}\right\rangle_{L}$ are exactly the same (not only the first terms in the series expansion given above). This also happens for the initial states $\left|\psi_{0}\right\rangle_{L}$ and $\left|\xi_{0}\right\rangle_{L}$. This is because when considering independent damping $\gamma$, there is no difference in the behavior between the physical states in (3) -(5)), while if we use the $[4,1] \times[4,1]$ code (encoded with the same labels in each qubit), the unique logical states that will behave in the same way, which is a desirable feature, are $|01\rangle_{L}$ and $|10\rangle_{L}$.

Disregarding this, let us study the effect of non-local coding for a pair of qubits and the possibility of obtaining better results, particularly protection against ESD, than with the local coding considered in [20]. In order to see the effect of the proposed QEC protocol, we should compare the fidelities given above with the fidelities given by the initial (not encoded) corresponding states, and the fidelities obtained with the $[4,1] \times[4,1]$ code for a pair of qubits which are given by[20]

$$
\begin{aligned}
& \mathcal{F}_{\phi_{1}}=1-\frac{\gamma^{2}}{2}\left(8-3 \cos 2 \alpha-2 \cos ^{2} 2 \alpha\right)+O\left(\gamma^{3}\right), \\
& \mathcal{F}_{\zeta_{1}}=1-\frac{\gamma^{2}}{4}\left(15-3 \cos 2 \alpha-2 \sin ^{2} 2 \alpha \cos ^{2} \beta\right)+O\left(\gamma^{3}\right), \\
& \mathcal{F}_{\psi_{1}}=1-\gamma^{2}\left(4-\cos ^{2} 2 \alpha\right)+O\left(\gamma^{3}\right), \\
& \mathcal{F}_{\xi_{1}}=1-\frac{\gamma^{2}}{4}\left(9-3 \cos 2 \alpha-2 \sin ^{2} 2 \alpha \cos ^{2} \beta\right)+O\left(\gamma^{3}\right),
\end{aligned}
$$

where we use the subscript " 1 " to indicate the use of the $[4,1] \times[4,1]$ in both the fidelities and in the (coded) states $\left(|\varphi\rangle_{L_{1}}\right)$. For the uncoded states (8)-(11) we will similarly label the fidelities with the subscript "0", and these fidelities are given by

$$
\mathcal{F}_{\phi_{0}}=1-2 \gamma \cos ^{2} \alpha+\gamma^{2} \cos ^{2} \alpha,
$$




$$
\begin{aligned}
& \mathcal{F}_{\zeta_{0}}=1-\gamma \cos ^{4} \alpha-\frac{\gamma^{2}}{16} \sin ^{2} 2 \alpha+O\left(\gamma^{3}\right) \\
& \mathcal{F}_{\psi_{0}}=1-\gamma \\
& \mathcal{F}_{\xi_{0}}=1-\frac{\gamma}{2}\left(5+2 \cos 2 \alpha+\cos ^{2} 2 \alpha\right)+\frac{\gamma^{2}}{8} \cos ^{2} \alpha(3+5 \cos 2 \alpha)+O\left(\gamma^{3}\right)
\end{aligned}
$$

From Equations (16) $-(21)$ is easy to see that with the $[6,2]$ code, the loss of the fidelity is of the same order as for the $[4,1] \times[4,1]$ code, in both cases decreases as $\sim \gamma^{2}$, while the decrease is linear for the uncoded states (8)-(11). A first-sight difference between the $[6,2]$ code and the $[4,1] \times[4,1]$ is that the fidelity of the entangled states in the first case depends on the parameter $\beta$ already in the second term in the series expansion for the Bell-like states, meanwhile for the second case this dependence is weak [20].

Since the fidelity for the states $|\phi\rangle_{L}$ and $|\zeta\rangle_{L}$ (using the [6,2] code) is the same, let us analyze its behavior and compare it with the fidelities $\mathcal{F}_{\phi_{1}}$ and $\mathcal{F}_{\zeta_{1}}$. Even if $\mathcal{F}_{\phi}$ and $\mathcal{F}_{\zeta_{1}}$ depend on the parameter $\beta$ already in the term of the order of $\gamma^{2}$, the differences introduced by this parameter (which reach their maximum when $\alpha=\pi / 4$ ) are not very significant compared with the ones introduced by $\alpha$, and we will disregard this parameter, setting it to zero, in the following. In Fig. 1 we compare the (exact) fidelities under dissipation for the states $|\phi\rangle$ and $|\zeta\rangle$ uncoded, and for both codes. In (a) it is shown that when $\alpha=\pi / 6$ the fidelities do not differ too much between the states and codes. This difference is increased as $\alpha$ becomes larger, and around $\alpha=\pi / 4$, Fig. 1(b), the separable state $|\zeta\rangle_{L_{1}}$ achieves a higher fidelity than the Bell-state $|\phi\rangle$, and the fidelity for this state is roughly the same for both codes. The difference between $\mathcal{F}_{\phi}$ and $\mathcal{F}_{\zeta_{1}}$ remains more or less equal until $\alpha=\pi / 2$, but $\mathcal{F}_{\phi_{1}} \rightarrow \mathcal{F}_{\zeta_{1}}$ as $\alpha \rightarrow \pi / 2$. This is because the state $|\zeta\rangle_{L_{1}}$ has a high probability amplitude for the physical state $|0000\rangle$, which is not evolving (see the codewords in [20]).

Nevertheless, in order to see how the QEC protocol is working we should compare not only the results obtained with the different codes, but with the fidelities without making use of any QEC. The uncoded state $\left|\zeta_{0}\right\rangle$, given by Eq. (10), after damping will have such a high fidelity that both codes are practically useless (see Fig. 1(b)). However, in this paper we are concerned about preventing ESD, and for the Bell-like state $|\phi\rangle$ QEC seems to work fine in terms of the fidelities, and we can say that for moderately large damping parameter $(\gamma \leq 0.3)$ the fidelity obtained by local coding will be higher or equal (in around $\alpha=\pi / 4$ ) than the 

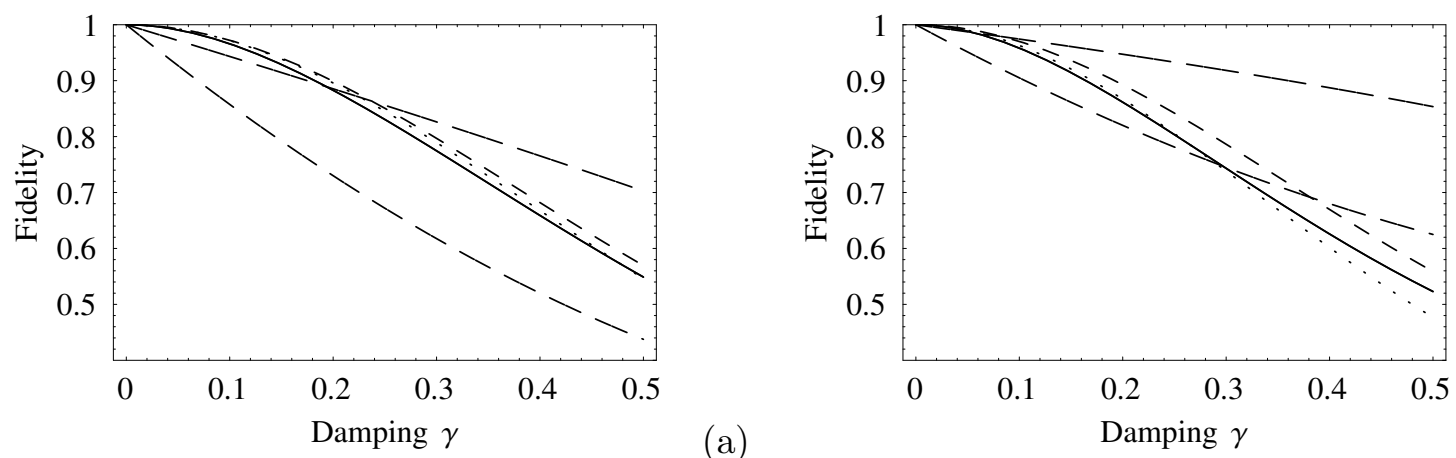

FIG. 1: Plot of the fidelities against the damping parameter $\gamma$, for the state $|\phi\rangle$, without QEC (dot-dashed), after recovering for the $[4,1] \times[4,1]$ code (dotted) and the code $[6,2]$ (continuous), and for the separable state $|\zeta\rangle$, without QEC (dot-dot-dashed), after recovering for the $[4,1] \times[4,1]$ code (dashed) and the code [6,2] (continuous), when (a) $\alpha=\pi / 6, \beta=0$ and (b) $\alpha=\pi / 4, \beta=0$.

one obtained by non-local coding. As was pointed out in [20], the fidelity is insufficient to characterize the remaining entanglement. Therefore we will study the concurrence as well. The analytic expressions of the concurrence are cumbersome and therefore we only present the exact, numerical plots shown in Fig. 2, where the concurrences are almost the same for values of $\gamma$ where it is worth to apply QEC. It is important to notice that even for non-local QEC, the protocol will introduce ESD when is not present in the uncoded state. For example, it is known that for the Bell-like states (8) the entanglement disappears under dissipation only when $\gamma=1$ if $|\cos \alpha| \leq|\sin \alpha|$.[8] However, in Fig. 2(b) is easy to see that this is not true when we use either of the QEC protocols. Moreover, we do not find any significative improvement by applying the $[6,2]$ code when comparing with the results presented in [20] for this states if we consider both, the fidelity and the concurrence.

As a final step we will do a similar analysis for the states $|\psi\rangle$ and $|\xi\rangle$, and in this case the differences between the fidelities are more notable, $\mathcal{F}_{\xi_{1}}$ being the higher and $\mathcal{F}_{\psi_{1}}$ being the lower in general, meanwhile for the $[6,2]$ code the fidelity $\mathcal{F}_{\psi}=\mathcal{F}_{\xi}$ is between them. As $\alpha \rightarrow \pi / 2$ the fidelities are roughly the same with the value, up to second order of $\gamma$, given by $1-3 \gamma^{2}$, being in this case the one for non-local coding a little higher than the other two. In Fig. 3 we plot the exact fidelities for this states for $\beta=0$ and (a) $\alpha=\pi / 4$, (b) $\alpha=\pi / 6$. We can conclude that, for this particular Bell-like state, encoding both qubits together leads 

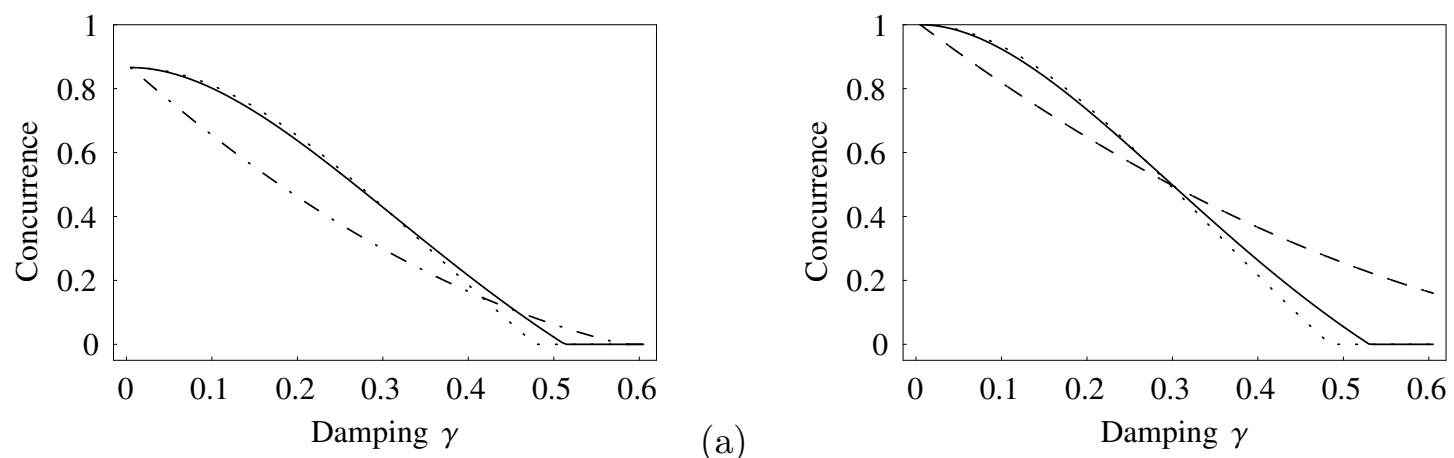

FIG. 2: Plot of the concurrences against the damping parameter $\gamma$, for the state $|\phi\rangle$, without QEC (dot-dashed), after recovering for the $[4,1] \times[4,1]$ code (dotted) and the code $[6,2]$ (continuous), when (a) $\alpha=\pi / 6, \beta=0$ and (b) $\alpha=\pi / 4, \beta=0$.

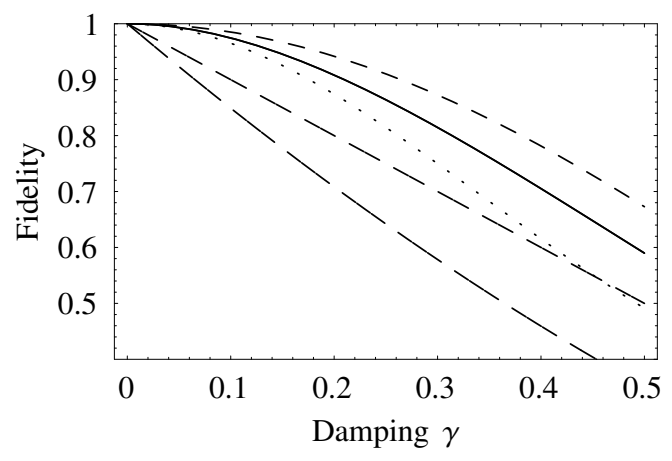

(a)

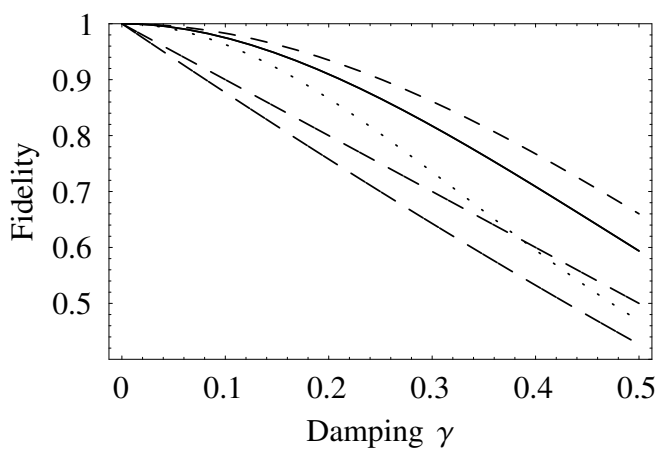

FIG. 3: Plot of the fidelities against the damping parameter $\gamma$, for the state $|\psi\rangle$, without QEC (dot-dashed), after recovering for the $[4,1] \times[4,1]$ code (dotted) and the code $[6,2]$ (continuous), and for the separable state $|\xi\rangle$, without QEC (dot-dot-dashed), after recovering for the $[4,1] \times[4,1]$ code (dashed) and the code [6,2] (continuous), when (a) $\alpha=\pi / 6, \beta=0$ and (b) $\alpha=\pi / 4, \beta=0$.

to a significative higher fidelity than encoding separately for certain values of $\alpha$ and $\beta$, this in contrast with the previous example. In summary, we can say that in terms of the fidelity the non-local QEC protocol presented here leads to better results in this case.

Now let us complement our study by means of the concurrence plotted in Fig. 4. By this figure we see that the non-local encoding will also leads to better results for the concurrence. Nevertheless, as it was pointed out in our previous work [20] the differences between the codes is more quantitative than qualitative, since for large values of $\gamma$ the coded state $|\psi\rangle$ loses its entanglement in a finite time, while the uncoded state loses it asymptotically, which 

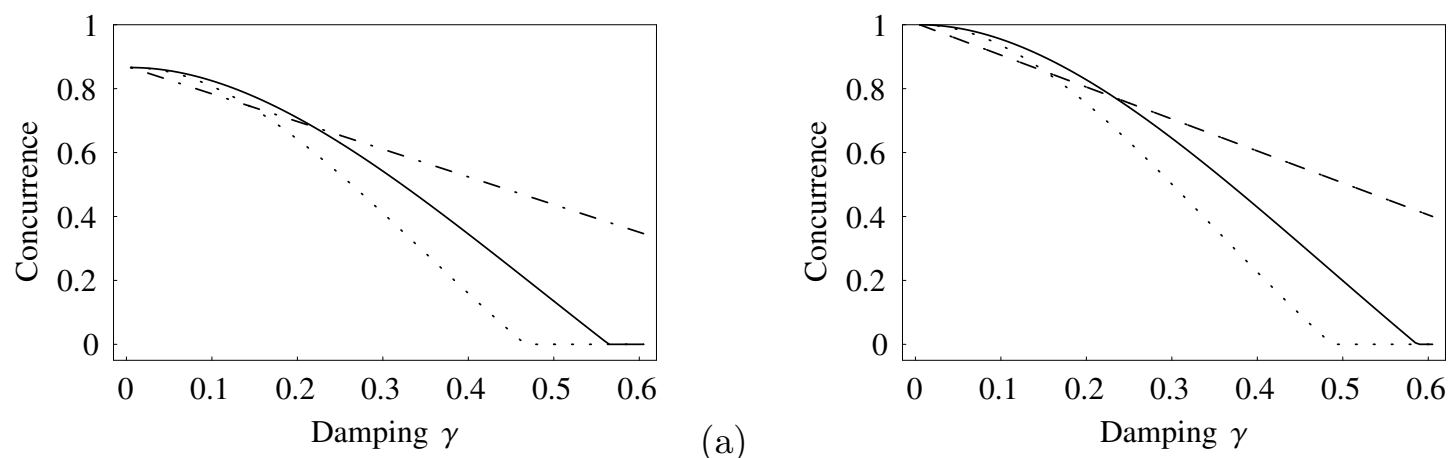

FIG. 4: Plot of the concurrences against the damping parameter $\gamma$, for the state $|\psi\rangle$, without QEC (dot-dashed), after recovering for the $[4,1] \times[4,1]$ code (dotted) and the code $[6,2]$ (continuous), when (a) $\alpha=\pi / 6, \beta=0$ and (b) $\alpha=\pi / 4, \beta=0$.

means that this QEC protocol will also introduce ESD to states that originally (uncoded) do not succumb to it. An explanation for this behavior is given in [21]. The asymptotic state under dissipation for the two uncoded states is the vacuum state $|00\rangle\langle 00|$ which is a pure separable state. Therefore it lies on the border between the sets of inseparable and separable states. The asymptotic (logical) state obtained using QEC corresponds to the vacuum state after applying the correction protocol, and with the two protocols considered these will be separable mixed states. These states lie inside the set of separable states. Hence, a continuous evolution from an initial entangled state $(\gamma=0)$ to the asymptotic state $(\gamma=1)$ must hence cross the border between inseparable to separable states for a finite amount of loss. However, for small values of $\gamma$ both the fidelity and concurrence achieved by non-local coding are higher than when using local coding for this Bell-like state.

\section{CONCLUSIONS}

With some examples we have studied the possibility of protecting two-qubit Bell-like states making use of a non-local code, we also have compared the results with previous obtained with some local coding [20]. We can conclude that for the Bell-like states $|\phi\rangle$ the non-local QEC protocol presented here does not lead to significant better results in terms of either, the fidelity and the concurrence, while for the Bell-like states labeled by $|\psi\rangle$ the results obtained for non-local coding are significantly better in comparison with local 
coding. However, this comparison is not completely fair because the local code, used here, is employing eight qubits in total and is able to correct some two-qubit errors (when each error is in different logical qubit) whereas the non-local code, due to its shorter length, can only correct a single error.

However, as was pointed out in [20], the implementation of a [6, 2] code requires creation of entanglement over the same physical distances as the entangled qubits exist over. Yet, qualitatively the results are the same, that is, QEC can delay ESD but it can also cause it for states that, uncoded, are not disentangled in a finite time. This behavior can be understood in terms of the asymptotic state formalism [21], which suggest that, in general, QEC will produce ESD in the logical qubits for any entangled state.

\section{Acknowledgments}

The authors thank Dr. Marcelo Terra Cunha for insightful comments. This work was supported by the Swedish Foundation for International Cooperation in Research and Higher Education (STINT), the Swedish Research Council (VR), and the Swedish Foundation for Strategic Research (SSF).

[1] M. Nielsen and I. Chuang, Quantum Computation and Quantum Information (Cambridge University Press, 2000).

[2] K. Życzkowski et al., Phys. Rev. A 65 (2001) 012101.

[3] T. Yu and J. H. Eberly, Opt. Commun. 264 (2006) 393-397.

[4] T. Yu and J. H. Eberly, Phys. Rev. Lett. 93 (2004) 140404.

[5] T. Yu and J. H. Eberly, Quantum Inf. Comp. 7 (2007) 459-468.

[6] L. Jakóbczyk and A. Jamróz, Phys. Lett. A 333 (2004) 35-45; M. F. Santos et al., Phys. Rev. A 73 (2006) 040305(R); T. Yu and J. H. Eberly, Phys. Rev. Lett. 97 (2006) 140403.

[7] J. H. Eberly and T. Yu, Science 316 (2007) 555-557.

[8] M. P. Almeida et al., Science 316 (2007) 579-582.

[9] G. M. Palma et al., in Proc. R. Soc. London, Ser A: Mathematical, Physical and Engineering Sciences, 8 March (JSTOR Press, London, 1996), pp. 567-584; I. L. Chuang and Y. Yamamoto, 
Phys. Rev. Lett. 76 (1996) 4281-4284; P. Zanardi and M. Rasetti, Phys. Rev. Lett. 79 (1997) 3306-3309; L.-M. Duan and G.-C. Guo, Phys. Rev. Lett. 79 (1997) 1953-1956.

[10] P. W. Shor, Phys. Rev. A 52 (1995) R2493-R2496.

[11] A. M. Steane, Phys. Rev. Lett. 77 (1996) 793-797.

[12] R. Laflamme et al., Phys. Rev. Lett. 77 (1996) 198-201.

[13] C. H. Bennett et al., Phys. Rev. A 54 (1996) 3824-3851.

[14] A. Ekert and C. Macchiavello, Phys. Rev. Lett. 77 (1996) 2585-2588; E. Knill and R. Laflamme, Phys. Rev. A 55 (1997) 900-911.

[15] D. Gottesman, Phys. Rev. A 54 (1996) 1862-1868; A. R. Calderbank et al., Phys. Rev. Lett. 78 (1997) 405-408.

[16] M. B. Plenio et al., Phys. Rev. A 55 (1997) 67-71.

[17] D. W. Leung et al., Phys. Rev. A 56 (1997) 2567-2573.

[18] A. S. Fletcher et al., Phys. Rev. A 77 (2008) 012320.

[19] A. S. Fletcher et al., Channel-Adapted Quantum Error Correction for the Amplitude Damping Channel, quant-ph/0710.1052.

[20] I. Sainz and G. Björk, Phys. Rev. A. 77 (2008) 052307.

[21] M. O. Terra Cunha, New J. Phys. 9 (2007) 237. 